\title{
Numerical Study to Improve the Back Interface Contact of CZTSSe Solar Cells by Oxygen-doped $\mathrm{Mo}\left(\mathrm{Se}_{1-\mathrm{x}}, \mathrm{O}_{\mathrm{x}}\right)_{2}$
}

Caixia Zhang ${ }^{1,2 *}$, Zhiyuan Yang ${ }^{1}$, Hui Deng ${ }^{1}$, Qiong Yan ${ }^{1,4}$, Weihao Xie ${ }^{1}$, Quanzhen Sun ${ }^{1}$, Xiaowei Sheng ${ }^{3}$, Shuying Cheng ${ }^{1,2 *}$

1. College of Physics and Information Engineering, and Institute of Micro-Nano Devices and Solar Cells, Fuzhou University, Fuzhou, 350108, P.R.China

2. Jiangsu Collaborative Innovation Center of Photovoltaic Science and Engineering, Changzhou, 213164, P.R.China

3. College of Physics and Electronic Information, Department of Physics, Anhui Normal University, Wuhu, 241000, P. R. China

4. Key Laboratory of Green Perovskites Application of Fujian Province Universities, Fujian Jiangxia University, Fuzhou, 350108, PR China *corresponding author email: zhangcx@fzu.edu.cn; sycheng@fzu.edu.cn.
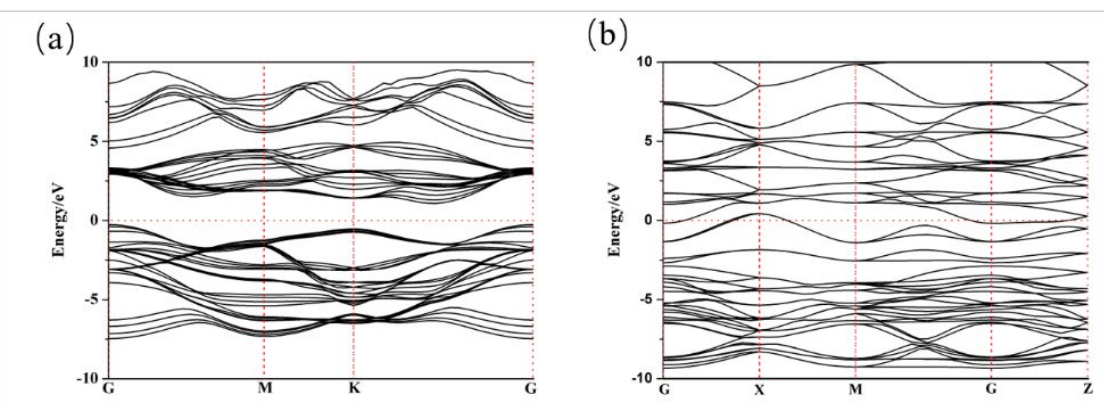

Fig. S1. The calculated band structures of (a) $\mathrm{MoSe} 2$ and (b)MoO2

Calculation details:

The band structures of $\mathrm{MoSe}_{2}$ and $\mathrm{MoO}_{2}$ reported herein were calculated using the Vienna ab initio simulation package (vasp) based on density functional theory ${ }^{1-2}$. The generalized gradient approximation (GGA) parametrization of Perdew-BurkeErnzerhof (PBE) was used to optimize the geometric structure of $\mathrm{MoSe}_{2}$ and $\mathrm{MoO}_{2}$ crystals. To include the electron-ion interactions, a planewave basis set with the projector augmented plane-wave method (PAW) was used. To find more accurate band gap values, we performed the standard Heyd-ScuseriaErnzerhof (HSE06) hybrid functional calculations with a fraction of the exact exchange of 0.25 , and a screening parameter of $0.2 \mathrm{~A}^{-1}$, which has proven to be a promising tool for accurate determination of the fundamental band gaps. The kinetic energy cutoff was taken as $460 \mathrm{eV}$ for the plane-wave basis set, while Gammapoint centered $k$-point grids of $10 \times 10 \times 1$ and $6 \times 5 \times 5$ were used for Brillouin zone integration of 
$\mathrm{MoSe}_{2}$ and $\mathrm{MoO}_{2}$ unit cell, respectively. The atomic positions were fully relaxed to minimize the forces to $0.005 \mathrm{eV} / \mathrm{A}$ or less. The convergence criterion for energy was set to less than $10^{-5} \mathrm{eV}$ between two consecutive steps in the self-consistent field calculations. Further increase in the cutoff value and $k$-point number did not lead to any noticeable changes in the eigenvalues.

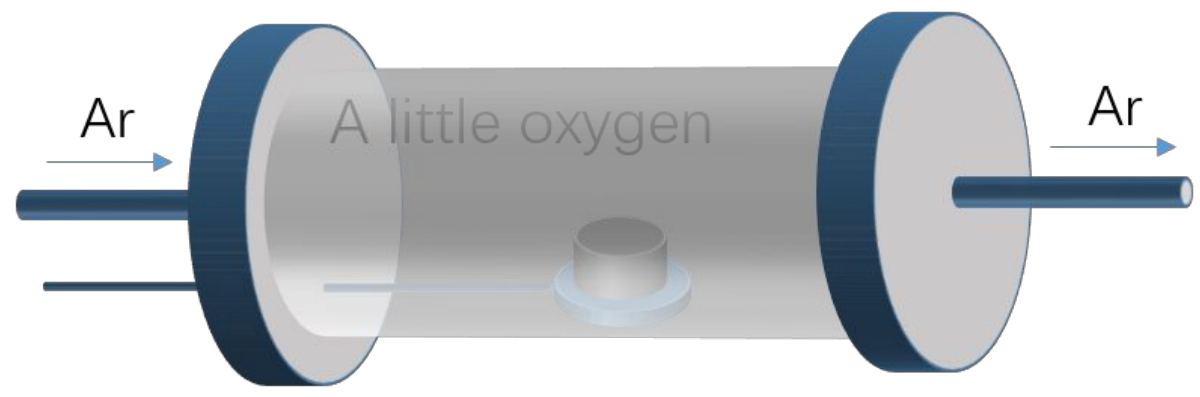

Fig. S2. Schematic diagram of selenization

Experimental details :

The process was carried out in a rapid thermal processer (RTP) with a ramp-up rate at $9^{\circ} \mathrm{C} / \mathrm{s}$ under dynamic nitrogen flow of $80 \mathrm{~mL} / \mathrm{min}$, using a graphite box containing $500 \mathrm{mg}$ Se particles. Before the above process, we carry out five operations of vacuuming and filling with $\mathrm{N}_{2}$ gas to ensure that there is a small amount of $\mathrm{O}_{2}$ in the RTP cavity

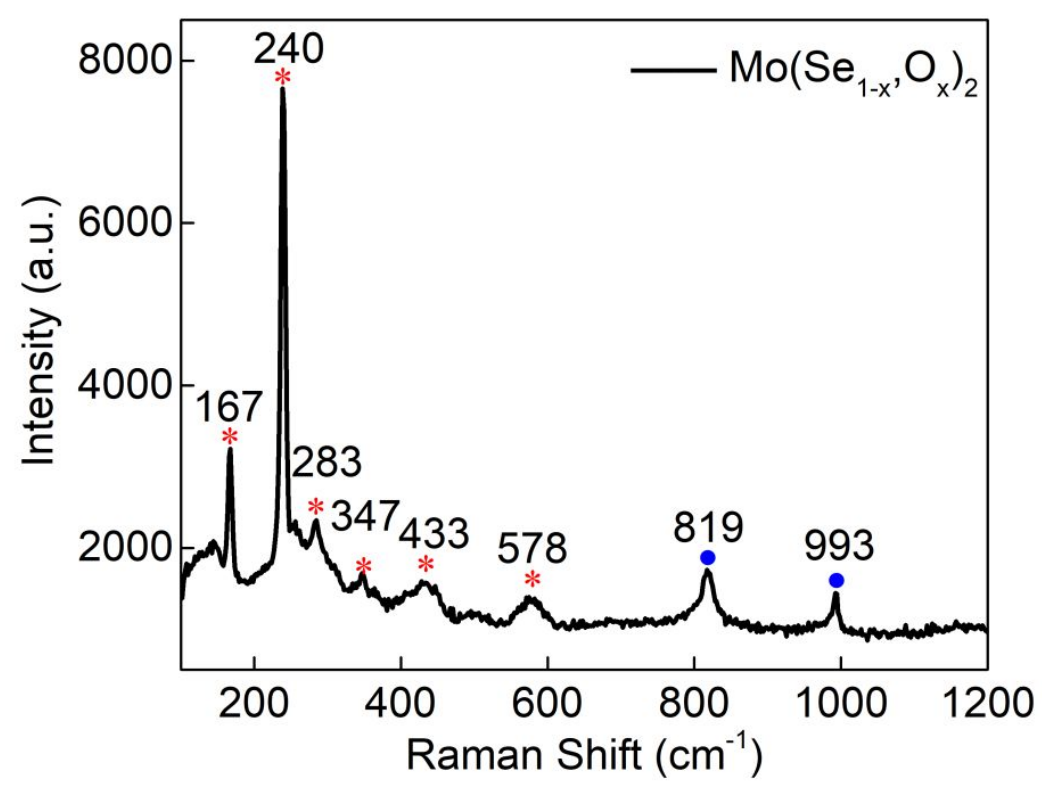

Fig. S3. Raman spectra (532 $\mathrm{nm}$ excitation wavelength) of $\mathrm{Mo}\left(\mathrm{Se}_{1-\mathrm{x}}, \mathrm{O}_{\mathrm{x}}\right)_{2}$ layer

Fig. S3 exhibits the Raman graph obtained from the experiment. In the figure, the peak marked by 
the red asterisk is in agreement with the reported Raman spectra of $\mathrm{MoSe}_{2}$. The peaks at $167 \mathrm{~cm}^{-1}$, $240 \mathrm{~cm}^{-1}$, and $283 \mathrm{~cm}^{-1}$ correspond to the $\mathrm{E}_{1 \mathrm{~g}}, \mathrm{~A}_{1 \mathrm{~g}}$, and $\mathrm{E}_{2 \mathrm{~g}}^{1}$ Raman modes of $\mathrm{MoSe}_{2}$, respectively ${ }^{3}$. The peaks at $347 \mathrm{~cm}^{-1}, 433 \mathrm{~cm}^{-1}$ and $578 \mathrm{~cm}^{-1}$ have been reported to exist in $\mathrm{MoSe}_{2}{ }^{4}$. The Raman peak at $993 \mathrm{~cm}^{-1}$ is assigned to the terminal oxygen $(\mathrm{Mo}=\mathrm{O})$ stretching mode. The peak at $819 \mathrm{~cm}^{-}$ ${ }^{1}$ can be assigned to the double coordinated oxygen (Mo-O-Mo) stretching mode ${ }^{5}$.

\section{References}

1. Kresse, G.; Hafner, J., Ab Initiomolecular-Dynamics Simulation of the Liquid-MetalAmorphous-Semiconductor Transition in Germanium. Physical Review B 1994, 49, 1425114269.

2. Hohenberg, P.; Kohn, W., Inhomogeneous Electron Gas. Physical Review 1964, 136, B864B871.

3. Wu, C.-T.; Hu, S.-Y.; Tiong, K.-K.; Lee, Y.-C., Anisotropic Effects in the Raman Scattering of Re-Doped 2h-Mose 2 Layered Semiconductors. Results in Physics 2017, 7, 4096-4100.

4. Nam, D.; Lee, J. U.; Cheong, H., Excitation Energy Dependent Raman Spectrum of Mose 2 . Sci Rep 2015, 5, 17113.

5. Wang, T.; Li, J.; Zhao, G., Synthesis of $\mathrm{Mos}_{2}$ and $\mathrm{MoO}_{3}$ Hierarchical Nanostructures Using a Single-Source Molecular Precursor. Powder Technology 2014, 253, 347-351. 